\title{
Real Wages and the Cycle: The View from the Frequency Domain
}

\author{
Robert A. Hart* \\ Department of Economics, University of Stirling \\ James R. Malley \\ Ulrich Woitek \\ Department of Economics, University of Glasgow
}

March 30, 2001

\begin{abstract}
In the time domain, the observed cyclical behavior of the real wage hides a range of economic influences that give rise to cycles of differing lengths and amplitudes. This may serve to produce a distorted picture of wage cyclicality. Here, we employ frequency domain methods that allow us decompose wages into cyclical components and to assess the relative contribution of each component. These are discussed in relation to wages alone (the univariate case) and to wages in relation to production or employment-based measures of the cycle (multivariate). In the multivariate dimension, we derive methods for determining whether (i) wage and business cycles cohere (ii) lead-lag or contemporaneous relationships exist and (iii) the degree of coherency between wage and business cycles is time dependent. We establish that real wages are strongly procyclical and that the business cycle is the dominant associated influence.
\end{abstract}

JEL Classification: E32, J31

*Corresponding Author: Robert Hart, Department of Economics, University of Stirling, Stirling FK9 4LA, UK, email: r.a.hart@stir.ac.uk. 
... correcting for all of the measurement problems, estimation problems, and com-

position problems does not lead to a finding of systematically procyclical or coun-

tercyclical real wages.

Abraham and Haltiwanger (1995)

\section{Introduction}

Abraham and Haltiwanger reach the above conclusion via a comprehensive survey of the empirical literature together with their own thorough empirical analysis. The statement would appear to be particularly valid in respect of hourly wage rates (excluding overtime). ${ }^{1}$ The volume of research and the various angles of approach are such that the observed weak relationship between the wage and the cycle has reached the status of a stylized fact. There is, however, one important line of investigation that has been left unexplored and that should be addressed before definitive conclusions can be reached. The vast majority of existing studies are concerned with auto- and cross-covariances in the time domain. But the cyclical behavior of wages in the frequency domain may not only serve to cast fresh light on the subject area but also provide insights into the reasons for the tenuous time-wise correlations.

Consider three cycles of relatively short, medium and long amplitudes. Although by no means hard and fast or exhaustive representations, these might consist of (respectively) a wage contract cycle, a business cycle and a product cycle. Each type may associate systematically with the real wage. The relative strength and direction of the associations may differ, however. The start of a three-year United States wage contract, for example, may involve wage adjustments designed to correct for unforeseen economic events at the previous negotiation time point. This process may be expected to generate a mix of pro- and countercyclical wage effects through time depending on direction of deviations from expected outcomes. Additionally, the wage may respond positively to the longer business cycle. For instance, where compensation relates to marginal product, human capital investment may produce procyclical wages stemming from the fixity of the labor input. The wage may also associate positively with the product cycle. Top quality workers earning relatively high pay may be matched with new and innovative products with strong growth potential. As these products are eventually superseded by new innovations, they may subsequently be associated with the hire of relatively poorer quality and less well-remunerated workers.

Yet, all three cyclical effects will serve to condition a long time series of the real wage.

\footnotetext{
${ }^{1}$ Hourly wage earnings (including overtime) display somewhat more evidence of procyclicality for the obvious reason that overtime pay and hours are strongly procyclical. See Hart and Malley (2001) for an empirical analysis of real wage earnings broken down into (i) the wage rate, (ii) the overtime mark-up, (iii) and the proportion of workers working overtime.
} 
This gives rise to a series of critical questions. Which, if any, is the dominant frequency band acting on the cyclical behavior of the wage? If a given frequency dominates, what direction and strength of cyclicality does it exhibit? Does an association within a frequency band between the wage and the cycle represent a contemporaneous association or involve leads or lags? Does this association change over time? Pursuing such lines of enquiry leads to a more general question. Is the observed wage cyclicality in the frequency domain supportive of the general view arising from time series analysis or does it serve to modify that view? A seeming low correlation between the wage and a measure of the cycle may simply reflect the fact that the underlying time series is composed of frequency bands between the two variables that are of different amplitudes and timing. Separately, one or more bands may display strong evidence of a systematic cyclical relationship. Taken together, countervailing influences may serve to mask underlying patterns.

Due to the fact that interest in the cyclical behavior of economic variables has concentrated disproportionately on the time domain, this paper sets out to achieve two main objectives. First, it attempts to illustrate, in as transparent a way as possible, the similarities and differences between the time and frequency domain methodologies. Second, it applies the two approaches to the analysis of real wage cyclicality in the United States. Estimation is undertaken on annual US manufacturing data for the period 1956-1997. Reflecting the previous literature, we adopt both industrial production and employment-based measures of the business cycle. We find that: (i) real wages are procyclical; (ii) the dominant frequency range is between 5 and 7 years, reflecting the relative importance of the business cycle; (iii) in line with the NBER business cycle chronology, the dominant cycle range changes through time to reflect relatively short business cycles prior to the early $1980 \mathrm{~s}$ and longer thereafter; (iv) the strength of the finding in (iii) is in part dependent on the use of wages deflated by a consumer price index rather than a producer price index; (v) incorporating a productionbased measure of the cycle, real wages and the cycle are contemporaneously procyclical; and (vi) incorporating an employment-based measure of the cycle, the cycle displays either a phased lag or an acyclical co-movement with the real wage.

Section 2 sets out the key concepts and principles involved in transforming a single (univariate) time-series from the time to the frequency domain. The spectrum measures the marginal contribution of each wave to the overall variance of the time-series, and is a function of the frequency of each wave. Therefore, to determine the length of the dominant cycle in a particular series, it is necessary to search the spectrum between the endpoints of the entire frequency interval and select the cycle that explains the greatest portion of the total variance of the series. Finally, it is shown how to re-map back to the time-domain to determine the number of years that it takes the cycle to reach peak-to-peak. This is possible since, as mentioned above, each of the individual spectra are a function of the frequency of each 
wave. The clear value-added in this context is that spectral analysis allows a decomposition of a series into cyclical components defined over multiple frequency bands. In time-domain analysis, by contrast, considerations relating to multiple frequencies are simply ignored.

Section 3 extends the analysis of Section 2 to a multivariate context. Section 3.1 draws parallels between simple two-variable correlation and the analogous concept, the so-called coherency measure, in the frequency domain. This measure can be obtained from the crossspectrum of two time-series. It is shown that the simple correlation coefficient is a special case of the frequency measure in that the former is the constant across all frequencies. In Section 3.2 , a test of whether the coherency measure in a particular frequency band is significantly different from zero is developed. This test is based on empirical distributions since asymptotic distributions are difficult to derive in this context and more importantly - even if they were not - we will be conducting our empirical analysis using small samples. In Section 3.3 we show how the cross spectrum in the frequency domain, which is analogous to the cross correlation in the time domain, can be used to identify lead-lag relationships between two variables. We also discuss how the application of a standard non-parametric bootstrap procedure can be used to conduct hypothesis tests which allow us to categorize the relationship between two variables as (i) acyclical; (ii) contemporaneously pro- or countercyclical; (iii) leading pro- or countercyclical and (iv) lagging pro- or countercyclical.

Section 4 contains discussion comparing the results of applying both time- and frequencydomain methods to a number of measures of the real wage and the cycle. Here we examine the cyclical behaviour of filtered average hourly earning, $A$ and average earning excluding overtime, $W$, each deflated by consumer, $C p$ and producer, $P p$ prices. Industrial production, $Y$ and manufacturing employment, $N$ are employed as measures of the business cycle with the de-trending methods including the modified Baxter-King (Baxter and King, 1995; Woitek, 1998), the stochastic difference and the Hodrick-Prescott (Hodrick and Prescott, 1980) filters.

Additionally, to examine the issue of whether the degree of coherency between the wage and the cycle changes over time, we develop and apply a method to estimate time dependent spectra based on the Kalman filter. Section 5 concludes the paper and Section A contains the appendices. Appendix A.4 sets out the detailed results which underpin the summary tables reported in Section 4 while appendices A.1-A.2 contain technical details.

\section{Univariate Measure}

We begin by illustrating a central argument of this paper. In order to determine the length of the dominant cycle in a particular series, it is necessary to work with its spectral representation. Suppose that we set out to examine the cyclical characteristics of the stationary time-series plotted in the bottom graph in Figure 1. In fact, it comprises the sum of three 
cosine waves, $y_{1}, y_{2}$, and $y_{3}$, defined as

$$
\begin{aligned}
& y_{1}=2 \cos \left(\pi 0.1 t+\frac{\pi}{6}\right) \\
& y_{2}=1.5 \cos \left(\pi 0.09 t-\frac{\pi}{4}\right) \\
& y_{3}=0.7 \cos \left(\pi 0.2 t-\frac{\pi}{5.8}\right) .
\end{aligned}
$$

Figure 1: Aggregation of Harmonic Waves
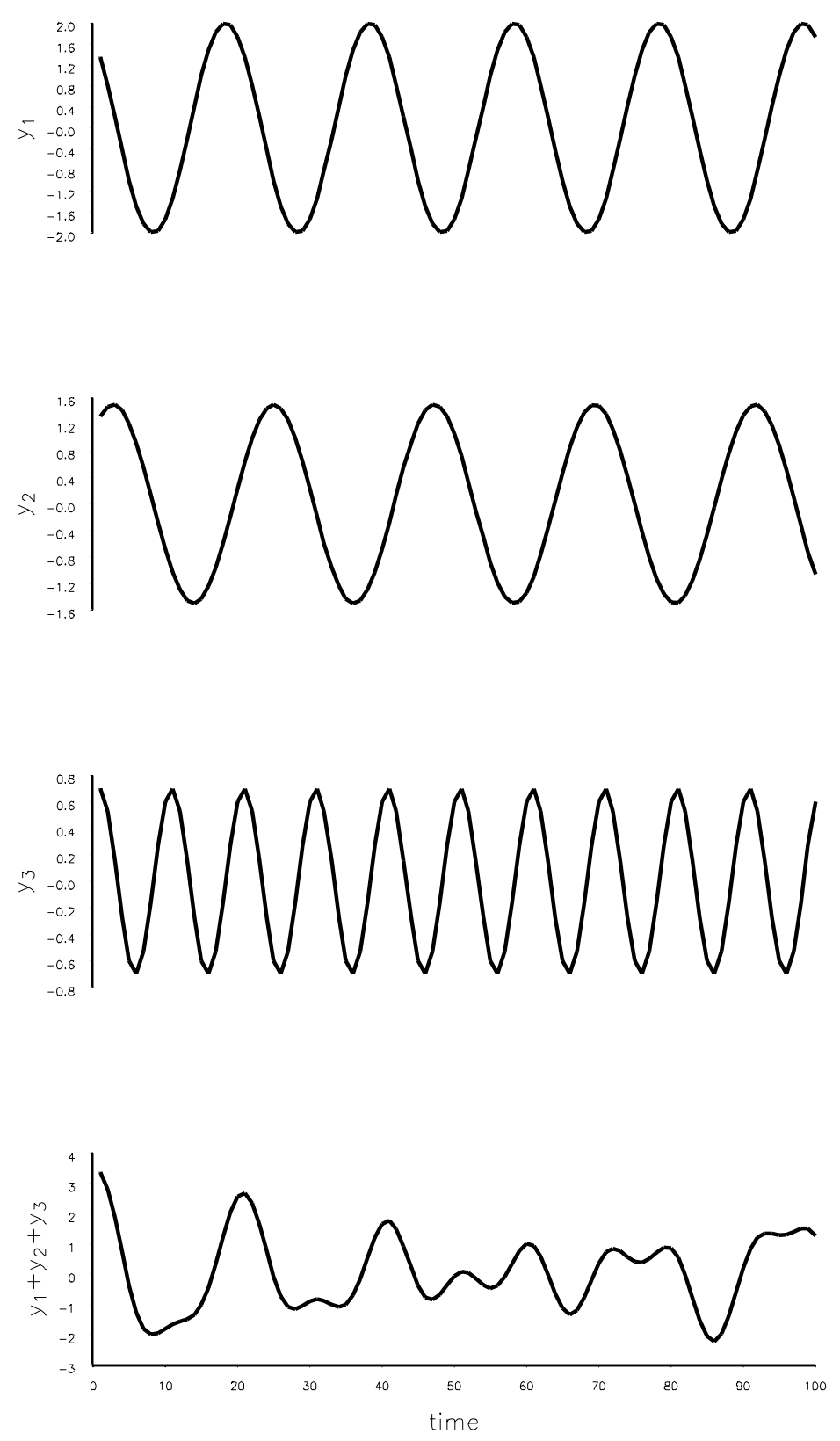
As illustrated in Figure 1, the three waves are harmonic waves of varying amplitudes while the aggregate time series follows a smoother time path. Clearly, describing the cyclical behavior of the latter would produce seriously misleading inferences if the adopted methodology were not capable of detecting the individual wave constituents.

Fourier transformation allows us to decompose a stationary series into superimposed harmonic waves. In other words, every stationary process/series $X_{t}$ has a spectral representation. It can be decomposed into superimposed harmonic waves with frequencies $\omega$ in the interval $[-\pi, \pi] .^{2}$ These waves have stochastic amplitudes and phases. The marginal contribution of each wave to the overall variance $\gamma_{x}(0)$ of the process is called the spectrum, and is a function of the frequency of the wave.

In empirical business cycle research, the predominant paradigm has been to examine auto- or cross-covariances in the time domain. ${ }^{3}$ The information on the cyclical structure contained in the autocovariance function can be transformed into frequency domain, revealing a more detailed picture. The spectrum of a process is defined as the Fourier transform of the autocovariance function $\gamma_{x}(\tau), \tau=0, \pm 1, \pm 2, \ldots$ :

$$
f_{x}(\omega)=\frac{1}{2 \pi} \sum_{\tau=-\infty}^{\infty} \gamma_{x}(\tau) e^{-i \omega \tau} ; \omega \in[-\pi, \pi] .
$$

Figure 2 illustrates the plot of a spectrum. ${ }^{4}$ The interpretation is like that of a probability density function; $f_{x}(\omega) d \omega$ is the part of the overall variance of $X_{t}$ which is due to the component with frequencies over the interval $[\omega, \omega+d \omega]$. The total area under the spectrum equals the process variance:

$$
\gamma_{x}(0)=\int_{-\pi}^{\pi} f_{x}(\omega) d \omega
$$

In other words, we can look at it as the plot of a decomposition of the variance against frequencies in the interval $[0, \pi] .{ }^{5}$ After normalising the spectrum using the variance $\gamma_{x}(0)$, the area under the curve from $\omega_{1}$ to $\omega_{2}$ in Figure 2 is (half) the share of total variance of $X_{t}$ which can be attributed to the composite of the waves in this range. Spectral analysis thus permits a natural decomposition of a series into cyclical components defined over frequency

\footnotetext{
${ }^{2} \mathrm{~A}$ cosine wave has a basic period of $2 \pi$. If we want to model a cycle with a length of 8 time units, we have to multiply $2 \pi$ with the frequency $\lambda=0.125$ to result in a peak-to-peak (trough-to-trough) distance for the function $\cos \omega t, \omega=2 \pi \lambda$, of 8 time units. The frequency $\lambda$ measures how often a cycle repeats itself per time unit (in our case, $1 / 8$ times), and is the reciprocal of the cycle length. The smallest cycle length is 2 time units, which corresponds to a maximum frequency $\lambda=0.5$, or, in terms of $\omega, \omega=\pi$.

${ }^{3}$ Widely cited examples are Kydland and Prescott (1990) and Backus and Kehoe (1992). For a recent exception, working in the frequency domain, see A'Hearn and Woitek (2001).

${ }^{4}$ We estimate parametric spectra, i.e. we start in the time domain by fitting autoregressive models to the data. A detailed explanation of the estimation procedure can be found in the Appendix, Section A.3.1.

${ }^{5}$ Since the spectrum is an even function, it is not necessary to plot it in the entire range $[-\pi, \pi]$.
} 
bands we are interested in. In terms of real wages, these may relate to 3 - year wage contract cycles, 5-7 year business cycles and still longer cycles generated, for instance, by product and process innovations.

Figure 2: Spectrum

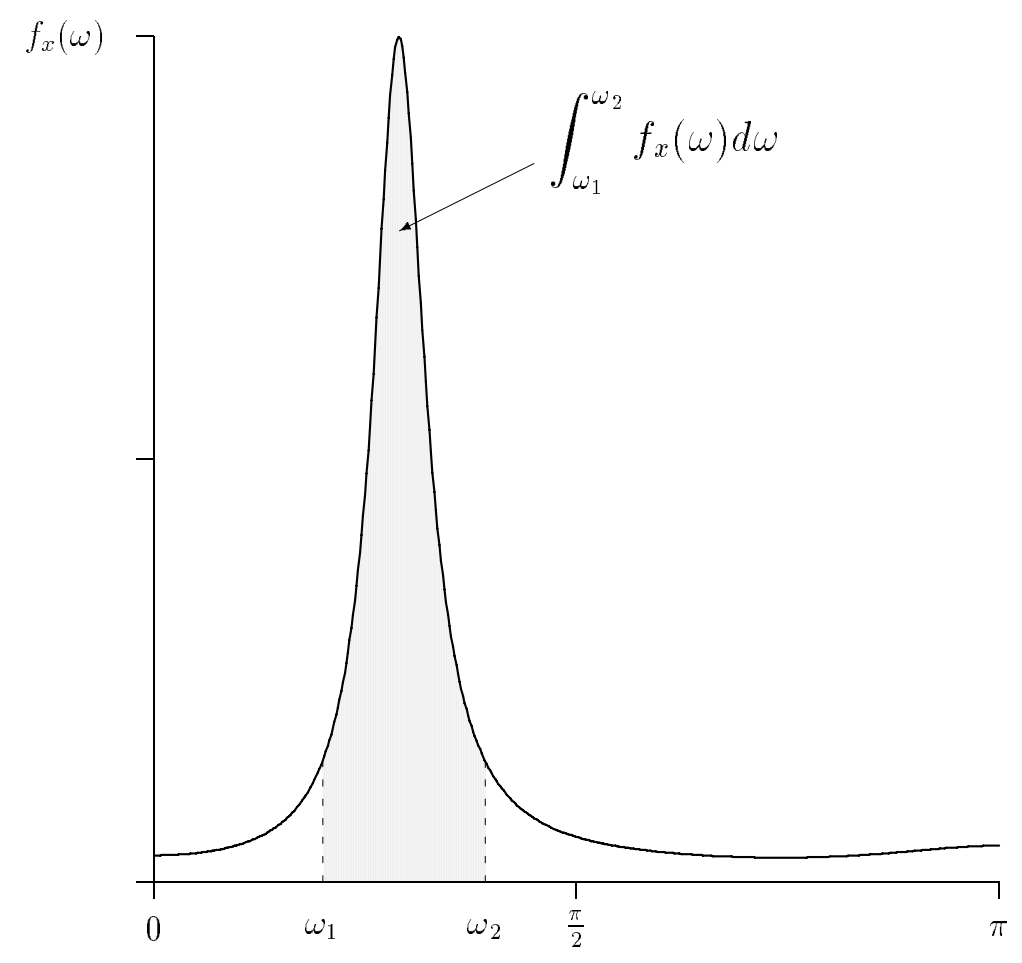

\section{Multivariate Measures}

A critical consideration has been omitted so far. The time series depicted at the bottom of Figure 1, may take on the appearance of a variable with strong cyclical characteristics but without an objective yardstick of what constitutes the economic cycle then definitive inferences are ruled out. In other words, does the univariate cyclical pattern correspond in some systematic way with a variable or variables that economists generally accept to be coincident, leading or lagging cyclical indicators? If the variable in Figure 1 is the real wage, then an obvious question to ask is how does its observed cyclical pattern co-vary with that of industrial production or aggregate employment. 


\subsection{Explained Variance}

To shed more light into the issues raised above in a bi-variate context, consider two stationary time series $X_{t}$ and $Y_{t}$. In the time domain, to assess the linear relationship between the two series, we start by looking at the simple linear regression model

$$
Y_{t}=a+b X_{t}+u_{t}, u_{t} \sim N I D\left(0, \sigma^{2}\right)
$$

The minimisation problem to be solved is given by

$$
\min _{a, b} \sigma^{2}=\mathrm{E}[Y_{t}-\underbrace{\left(a-b X_{t}\right)}_{\hat{Y}_{t}}]^{2},
$$

and as estimator for $b$ we obtain

$$
\hat{b}=\frac{\gamma_{y x}(0)}{\gamma_{x}(0)}
$$

i.e. the covariance between $X_{t}$ and $Y_{t}$ at lag zero, divided by the autocovariance of $X_{t}$ at lag zero (i.e. the variance). The following decompositions of the variance of $Y_{t}$ are useful:

$$
\gamma_{y}(0)=\gamma_{\hat{y}}(0)+\hat{\sigma}^{2}
$$

i.e. the variance of $Y_{t}$ can be decomposed into the variance of the part of $Y_{t}$ explained by the model in equation (3), and the residual variance. The variance of the "explained" part of $Y_{t}$, $\hat{Y}_{t}$, is given by

$$
\gamma_{\hat{y}}(0)=\hat{b}^{2} \gamma_{x}(0)
$$

hence,

$$
\gamma_{y}(0)=\hat{b}^{2} \gamma_{x}(0)+\hat{\sigma}^{2}
$$

The goodness of fit of the above model, $R^{2}$, can be calculated as the ratio of the explained variance to the overall variance:

$$
R^{2}=\frac{\gamma_{\hat{y}}(0)}{\gamma_{y}(0)}=\hat{b}^{2} \frac{\gamma_{x}(0)}{\gamma_{y}(0)}
$$


Inserting equation (7) into equation (6) gives

$$
\gamma_{y}(0)=R^{2} \gamma_{y}(0)+\hat{\sigma}^{2}
$$

In the frequency domain, we can derive a similar decomposition. We start by searching for the linear filter $B(L)$ which minimises the expression

$$
\sigma^{2}=\mathrm{E}[Y_{t}-\underbrace{B(L) X_{t}}_{\hat{Y}_{t}}]^{2} .
$$

It turns out that if we solve this problem in the frequency domain, the transfer function ${ }^{6}$ of the optimal filter $\hat{B}(L)$ is given by

$$
\hat{B}(\omega)=\frac{f_{y x}(\omega)}{f_{x}(\omega)}, \omega \in[-\pi, \pi]
$$

where $f_{x}(\omega)$ is the spectrum of the series $X_{t}$, and $f_{y x}(\omega)$ is the cross-spectrum for $Y_{t}$ and $X_{t}{ }^{7}$ If we decompose the spectrum of $Y_{t}$ into the part which can be explained by the filtered series $X_{t}$ and a residual spectrum, we obtain

$$
f_{y}(\omega)=f_{\hat{y}}(\omega)+f_{u}(\omega)
$$

where $f_{\hat{y}}(\omega)$ is given by

$$
f_{\hat{y}}(\omega)=|\hat{B}(\omega)|^{2} f_{x}(\omega)
$$

Hence, we can write equation (11) as

$$
\begin{aligned}
f_{y}(\omega) & =|\hat{B}(\omega)|^{2} f_{x}(\omega)+f_{u}(\omega)= \\
& =\frac{\left|f_{y x}(\omega)\right|^{2}}{f_{x}(\omega)}+f_{u}(\omega) .
\end{aligned}
$$

Again, the similarity to the procedure in time domain is obvious. In the next step, let us introduce a measure which assesses the degree of linear relationship between cylical compo-

\footnotetext{
${ }^{6}$ The transfer function models the effect of the filter to the series $X_{t}$, frequency by frequency.

${ }^{7}$ This is very similar to the expression in equation (5): the spectrum is the Fourier transform of the autocovariance function, and the cross-spectrum is the Fourier transform of the cross-covariance function:

$$
f_{y x}(\omega)=\frac{1}{2 \pi} \sum_{\tau=-\infty}^{\infty} \gamma_{y x}(\tau) e^{-i \omega \tau} ; \omega \in[-\pi, \pi]
$$
}


nents in the two series, frequency by frequency. This measure is the squared coherency $(s c)$, and is defined as

$$
s c(\omega)=\frac{\left|f_{y x}(\omega)\right|^{2}}{f_{x}(\omega) f_{y}(\omega)} ; \quad 0 \leq s c(\omega) \leq 1
$$

Using this expression, we can rewrite equation (12) as

$$
\begin{aligned}
f_{y}(\omega) & =\frac{\left|f_{y x}(\omega)\right|^{2}}{f_{x}(\omega) f_{y}(\omega)} f_{y}(\omega)+f_{u}(\omega)= \\
& =s c(\omega) f_{y}(\omega)+f_{u}(\omega) .
\end{aligned}
$$

To proceed, we need the result that the area under the spectrum is equal to the variance of the series $^{8}$, we can rewrite equation (8) as

$$
\underbrace{\int_{-\pi}^{\pi} f_{y}(\omega) d \omega}_{\gamma_{y}(0)}=\underbrace{\int_{-\pi}^{\pi} R^{2} f_{y}(\omega) d \omega}_{\text {"explained” variance }}+\hat{\sigma}^{2} .
$$

Integrating equation (14) over the frequency band $[-\pi, \pi]$ gives

$$
\underbrace{\int_{-\pi}^{\pi} f_{y}(\omega) d \omega}_{\gamma_{y}(0)}=\underbrace{\int_{-\pi}^{\pi} s c(\omega) f_{y}(\omega) d \omega}_{\text {"explained" variance }}+\underbrace{\int_{-\pi}^{\pi} f_{u}(\omega) d \omega}_{\tilde{\sigma}^{2}} .
$$

The first term on the right in equation $\left(14^{\prime}\right)$ is the product of squared coherency between $X_{t}$ and $Y_{t}$ and the spectrum of $Y_{t}$; the second term is white noise. This equality holds for every frequency band $\left[\omega_{1}, \omega_{2}\right]$. We can plot total variance (the area under the spectrum) and explained variance as shown in Figure 3. Comparing the area under the spectrum of the explained component to the area under $Y$ 's spectrum in a frequency interval $\left[\omega_{1}, \omega_{2}\right]$ yields a measure of the explanatory power of $X$, analogous to an $R^{2}$ in the time domain.

\footnotetext{
${ }^{8}$ See equation $(2)$
} 
Figure 3: Explained Variance

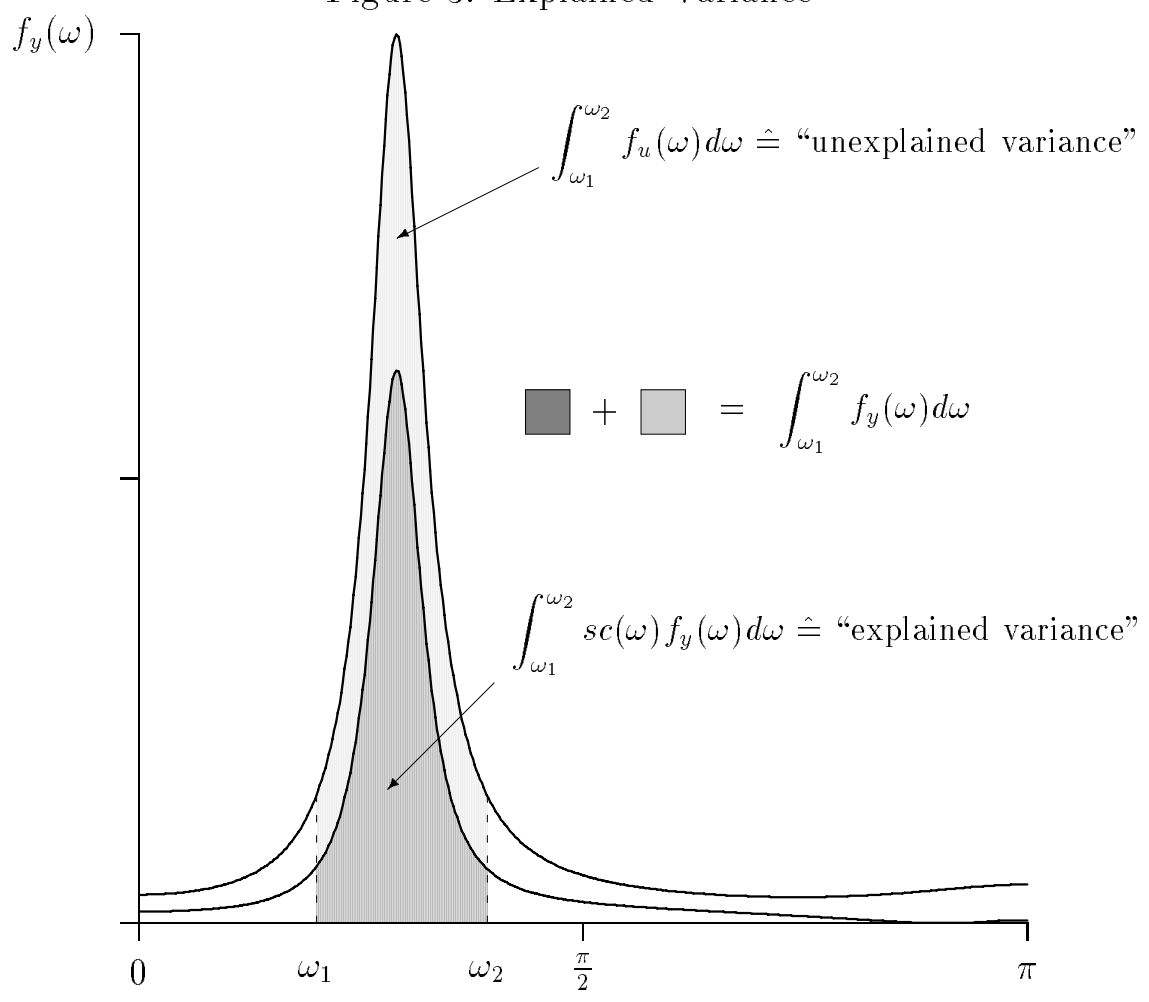

Comparing equations $\left(8^{\prime}\right)$ and $\left(14^{\prime}\right)$ shows both the similarities and differences between calculating measures of linear relationship in the time or frequency domain. In both cases, it boils down to decomposing the variance of a series into an "explained" and an "unexplained" part. In the frequency domain, however, this decomposition is done frequency by frequency. There are several reasons why such a measure might be more interesting. (i) The series might be dominated by cycles in a certain frequency. For example, while the peak-to-peak and trough-to-trough business cycles varied considerably in length, the mean post-war cycle lengths taken from the NBER reference dates and based on 9 cycles between 1945 and 1991 is 61 months. Do such business cycles, of between 5 and 7 years, exhibit more or less association with real wage movements compared to shorter (3-5 years) or longer (7-10) years? (ii) The correlation between cyclical components in the two series will be the same for all frequencies as a special case only, i.e. from equation $\left(8^{\prime}\right)$ we can see that $R^{2}$ is not a function of $\omega$, whereas in equation $\left(14^{\prime}\right), s c$ is a function of $\omega$. If the real wage associates with a given type of cycle then we would expect systematic relationships between changes in amplitudes of wage and cycle measures. 


\subsection{Testing the Significance of Explained Variance}

As discussed above, unlike in the time domain, with spectral analysis we obtain a different measure of linear association at every frequency. Therefore, we require a method which not only allows us to determine where the ratio of explained to unexplained variance is at a maximum, but also to determine whether, at this point, the explained variance is significant. ${ }^{9}$

Denoting explained variance as $\rho_{Y X}$, we next briefly describe a test to determine whether $\rho_{Y X}$ between two series $Y$ and $X$ in the relevant frequency band $\left[\omega_{1}, \omega_{2}\right]$ is significantly different from zero. ${ }^{10}$ As a first step, we fit AR models to $Y$ and $X$. We use the AR parameters to simulate the model under the Null hypothesis (i.e. no interaction between the two series). As a result, we obtain a simulated series $\left(Y_{t}^{s} X_{t}^{s}\right)^{\prime}$, which has the univariate characteristics of the underlying data, but without interaction. Next we fit a VAR of fixed order to $\left(Y_{t}^{s} X_{t}^{s}\right)^{\prime}$ and calculate $\rho_{X Y}^{s}$. These steps are repeated for $s=1, \ldots, 1000$. In this way, we obtain an empirical distribution of $\rho_{X Y}$ under the Null, conditional on the series we are examining. The critical values for each of the 24 models ( 4 real wages, 3 filters, output and employment) are displayed in Table 5 and 6 in Appendix A.4.

\subsection{Phase Shift}

Labor market phenomena like labor hoarding may introduce lags between, say, an upturn in production activity and its associated employment growth. Thus, observed cyclical relationships between the real wage and a production-based measure of the cycle may only be reflected via an employment-based measure after a lag, or phase-shift.

To identify the lead-lag relationship between the series $Y_{t}$ and $X_{t}$ in time domain, one would examine the cross correlations at lags $\tau= \pm 1, \pm 2, \ldots$, . Using the cross spectrum, this can be done frequency by frequency. The cross spectrum, which is the Fourier transform of the covariance function of $Y_{t}$ and $X_{t}$, is given by

$$
f_{y x}(\omega)=c_{y x}(\omega)-i q_{y x}(\omega),
$$

where $c_{y x}(\omega)$ is the cospectrum and $q_{y x}(\omega)$ is the quadrature spectrum. It can be used to derive the phase spectrum defined as

$$
\phi_{y x}(\omega)=-\arctan \left(q_{y x}(\omega) / c_{y x}(\omega)\right)
$$

\footnotetext{
${ }^{9}$ See Priestley (1981, p.705-706) for a similar the test of zero coherency for the classical spectral estimate, the periodogram.

${ }^{10}$ Since asymptotic distributions are extremely difficult to derive in this context, we construct an empirical distribution of $\rho_{X Y}$. More importantly, since we use relatively short time series, the asymptotics might not be valid anyway.
} 
The phase spectrum at frequency $\omega$ measures the lead of the cyclical component of $Y_{t}$ at this frequency over the corresponding component of $X_{t}$. It can be interpreted as the negative of the angle which would transform the component in $X_{t}$ into the best linear approximation of $Y_{t}$.

The phase spectrum as described by equation (15) is a bit difficult to interpret, due to discontinuous jumps when representing the phase spectrum on a linear scale (Koopmans, 1974, p.286). Instead, we present the results for the phase shift (at the frequency corresponding to a maximum in the real wage spectrum) on a circular scale. In addition to the point estimates, we also present confidence intervals for the phase shift.

Figure 4: Phase Shift

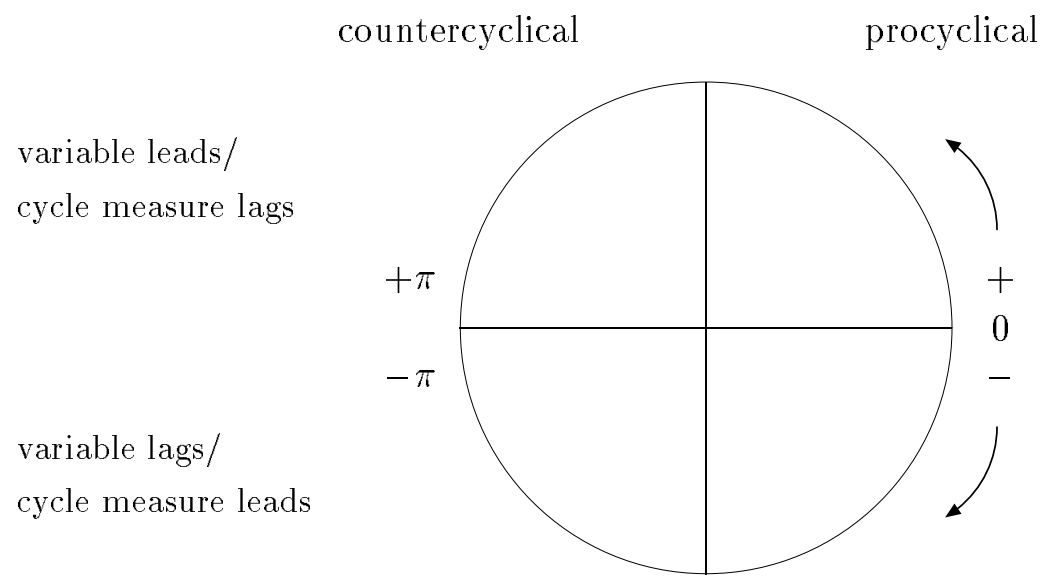

Presenting the phase shift on a circular scale leads to a straightforward interpretation of the confidence intervals (thick curves in Figures 6 and 7, Section A.4). ${ }^{11}$ The circle is divided into four regions: if the confidence interval covers the left half of the cycle, we interpret it as a significant counter-cyclical result. A confidence interval in the right half of the circle indicates procyclical movement. If the variable significantly leads the cycle measure, we would expect the confidence interval to be in the upper half of the circle. In the opposite case, we would expect it in the lower half. We interpret the case in which 0 is covered by the confidence interval as the cycles in the two series being in phase (i.e. "contemporaneously procyclical"). If $+\pi /-\pi$ is covered by the confidence interval, the two series are "contemporaneously countercyclical". If more than half the circle covered by the confidence interval, we term it acyclical, because it is impossible to derive information about the contemporaneous or

\footnotetext{
${ }^{11}$ To obtain the 90 per cent confidence intervals, a standard non-parametric bootstrap technique was applied (Efron and Tibshirani (1993)).
} 


\section{Applications to real wage cycles}

To provide a basis for comparison, based on U.S. annual data for the period 1959 to $1994^{12}$, we begin by showing in Table 1 estimated contemporaneous bivariate correlations between real wages and industrial production using filtered data. Table 1 estimates are based on both a production-based and an employment-based measure of the cycle. Our compensation measures comprise the earnings rate $A$ (which includes overtime), the wage rate $W$ (which excludes overtime) and two deflators, the producer price index $P p$ and the consumer price index $C p$. The results in Table 1 reveal that there is reasonable consistency in the estimates across the three chosen filters. In common with Abraham and Haltiwanger (1995) and others, wages and earnings in the time domain are found to display more procylicality when deflated by the $C p$ index. Moreover, in this latter case, cyclicality is enhanced when compensation includes the effects of overtime working. The employment-based cycle results are generally weaker than their production-based equivalents. Only $A / C p$ provides evidence of procyclicality. Taken together, these time series findings are mixed. Depending on how we classify the real wage, we find support for both procyclical and acyclical wage movements. This conclusion is unsurprising when viewed against the background of the existing literature.

Table 1: Real Wages and Business Cycles, Correlation Coefficients

\begin{tabular}{llrrrrrr}
\hline \hline & & \multicolumn{3}{c}{ Industrial Production } & \multicolumn{3}{c}{ Employment } \\
& & BKM & D & HP & BKM & D & HP \\
\hline$A / C p$ & $\tilde{\beta}$ & 0.55 & 0.58 & 0.57 & 0.29 & 0.34 & 0.25 \\
& $t_{h c}$ & 4.21 & 4.23 & 4.43 & 1.82 & 2.29 & 1.67 \\
$A / P p$ & $\tilde{\beta}$ & 0.21 & 0.16 & 0.17 & -0.13 & -0.07 & -0.01 \\
& $t_{h c}$ & 1.37 & 0.94 & 1.07 & -0.75 & -0.43 & -0.08 \\
$W / C p$ & $\hat{\tilde{\beta}}$ & 0.39 & 0.4 & 0.39 & 0.13 & 0.18 & 0.11 \\
& $t_{h c}$ & 2.71 & 2.60 & 2.66 & 0.77 & 1.13 & 0.69 \\
$W / P p$ & $\hat{\tilde{\beta}}$ & 0.12 & 0.04 & 0.05 & -0.22 & -0.17 & -0.09 \\
& $t_{h c}$ & 0.75 & 0.25 & 0.32 & -1.35 & -1.06 & -0.55 \\
\hline
\end{tabular}

Notes: (i) $A$ : average hourly earnings; $W$ : average hourly earnings excluding overtime; $P p$ : producer price index; $C p$ : consumer price index; (ii) BKM: modified Baxter-King filter; D: Difference filter; HP: Hodrick-Prescott filter.

\footnotetext{
${ }^{12}$ Note that the modified Baxter King filter requires that we drop three observations from both the start and the end of our sample.
} 
We next turn to the first of our frequency domain results in Table 2. The first two columns show univariate estimates pertaining only to the real wage variable. ${ }^{13}$ Our univariate spectral analysis results reveal that that the dominant cycle length is between 5 and 7.5 years for all measures of the real wage. Additionally, the relative contribution of these dominant cycles across real wage measures to explaining overall variation is between 33 and 57 percent. The next six columns present the results of the test developed in Section 3.2. That is, whether the coherency measure between real wages and the business cycle, based on both production and employment cycles, in a particular frequency band is significant. With the exception of the HP filter, all wage measures display uniformly significant co-movements in the 5-7 year range. ${ }^{14}$ Contrary to the time domain results in Table 1 , these findings are robust to the choice of (i) a consumer or a producer-based price deflator and (ii) a production or an employment measure of the cycle. While the 5-7 year cycle is dominant throughout, we note also that the shorter 3-5 year cycle is also generally significant. Results with respect to the long 7-10 year cycle are more patchy.

A summary of the results for estimating the phase shift, using the BKM filter, are shown in Table $3 .^{15}$ It is notable that irrespective of the real wage measure, these results indicate that there are no significant out of phase shifts in respect of the production-based cycle. In other words, the co-movements between real compensation and industrial production are unambiguously in phase or contemporaneously related. By contrast, the $N$ cycle is found either to lag real wage changes (using the producer price deflator) or to be acyclical (using the consumer price index). The most likely explanation for these differences in results between the two measures of the cycle is that employment is less cyclically sensitive than production. The phenomena of labor hoarding, long-term employment contracts and firm/union bargaining over job security may combine to produce low employment-output elasticities. ${ }^{16}$

\footnotetext{
${ }^{13}$ Note that this table is based on a search conducted over three frequency ranges: 7 -10 years (Juglar cycle), 5-7 years, and 3-5 years (Kitchin cycle).

${ }^{14}$ It is well established that the HP filter does not only cause serious distortions of the cyclical structure if applied to difference stationary series. For example, it has shown that there is a high probability of producing spurious correlations between independent random walk series (Harvey and Jaeger, 1993; King and Rebelo, 1993; Cogley and Nason, 1995). Additionally, the fact that the filter is not symmetric at the beginning and the end of the series leads to a distortion of the lead-lag relationship between the filtered series.

${ }^{15}$ For further details, see Figures 6 and 7 in Appendix A.4.

${ }^{16}$ In a review of four studies, Hamermesh (1993, Table 7.5) reports U.S. intermediate employment-output elasticities of between 0.37 and 0.43 and long-run elasticities between 0.50 and 0.92 . The last figure is something of an outlier; the mean long run elasticity 0.68 .
} 


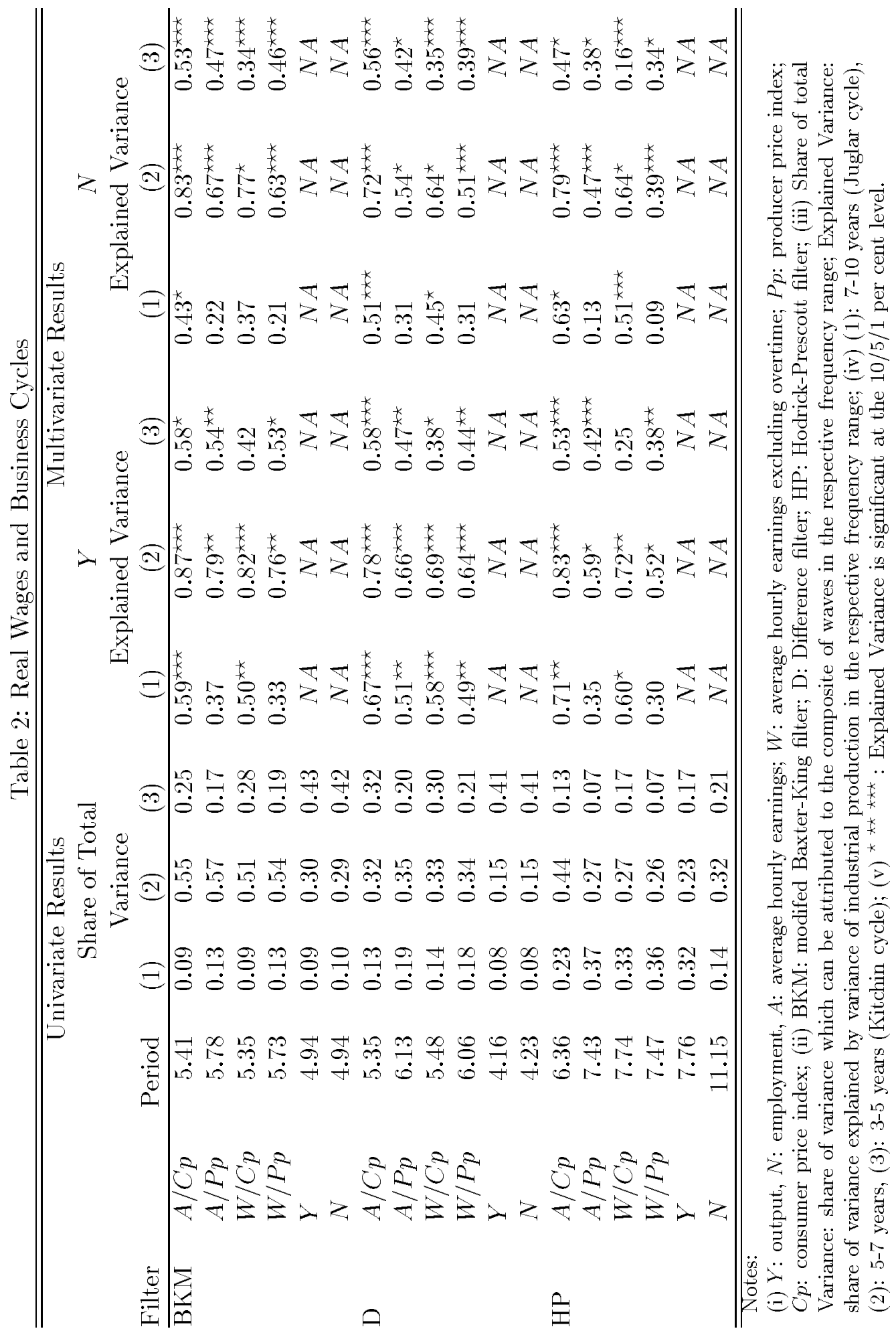




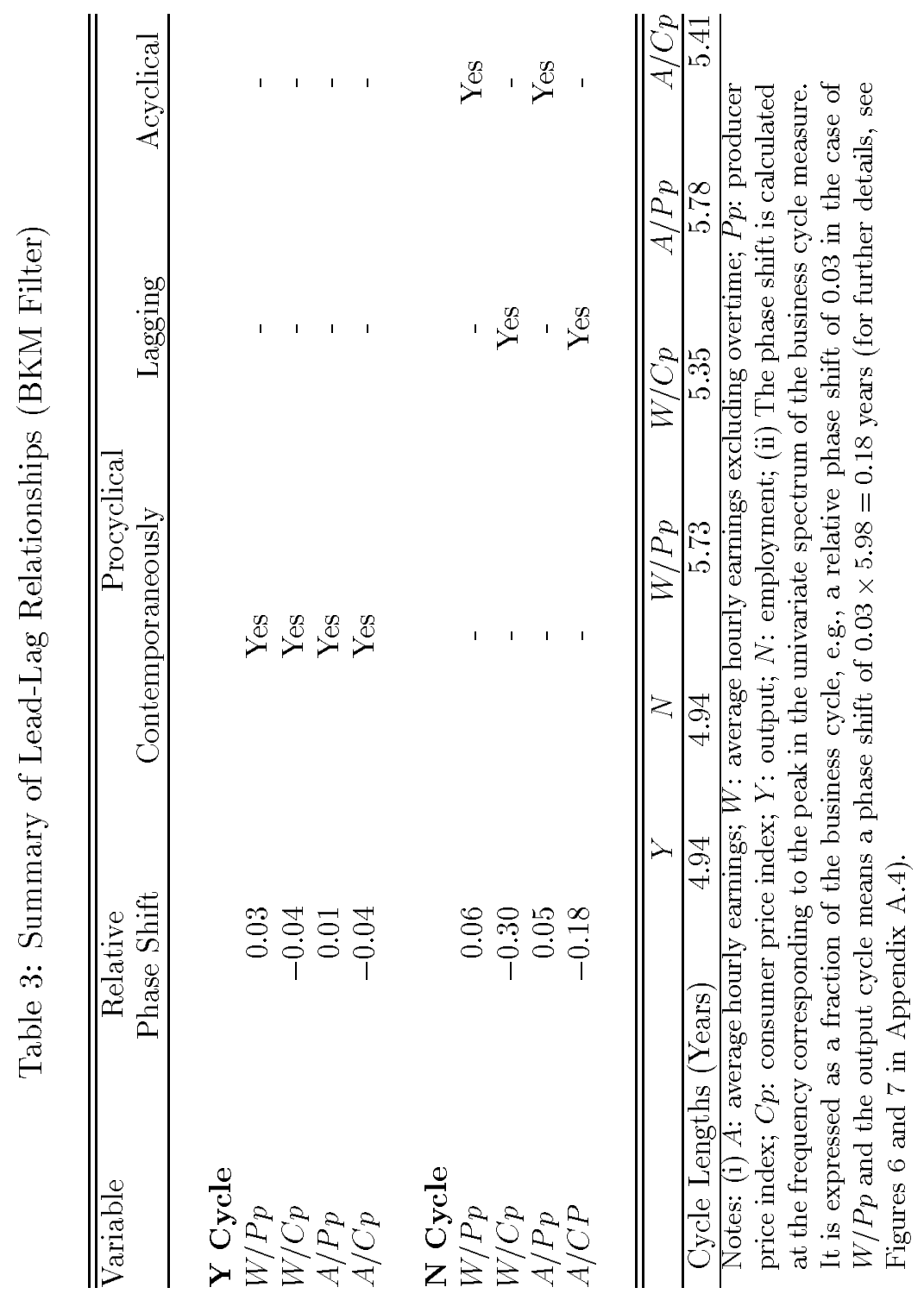


In general, our frequency domain findings point strongly towards a procyclical association between real compensation and the business cycle. But, in the post-war era there has been quite wide cyclical variation around this average. A critical question is whether the dominant frequency wage bands change through time in line with changes in the lengths of the business cycle. From the NBER chronology, the year 1981 represents a watershed between a period of relatively short peak-to-peak business cycles followed by a long cycle. Between December 1969 and July 1981 there were 3 cycles that averaged 3.9 years. The following cycle lasted 9 years, from July 1981 to July 1990. If the business cycle were a dominant influence on real wage movements then we would expect this considerable lengthening of cycle to be reflected in the observed cyclical wage patterns.

Accordingly, Table 4 shows the time dependent spectra ${ }^{17}$, dividing the $1969-1990$ period at 1981. We find that the $Y$ and $N$ cycles in the periods before 1981 are shorter and those after 1981 longer. If we take in the production cycle, the proportions of the total variance explained by the 3-5 and the 5-7 frequency bands fall, respectively, from 41 to 21 and from 39 to 35 percent. In contrast, the contribution of the 7-10 year cycle rises from 9 to 21 percent. These changes are matched by real wages deflated by the consumer price index. The short cycle (3-5 years) for $W / P c$ contributes 50 percent of the explained variance in the early period and this falls to 35 percent post-1981. Comparably, the percentage drops from 33 to 13 for the middle cycle (5-7 years). By contrast, the percentages rise from 7 to 22 percent for the long cycle (7-10 year). Wages and earnings deflated by the producer price index display similar cyclical re-weightings between the two periods though of somewhat lower magnitudes. For $W / P p$, there is a fall in the percentage contribution to variance from 040 to 0.25 in the short cycle, no change (at 0.26 ) in the middle cycle, and a rise from 0.28 to 0.38 in the long cycle.

\footnotetext{
${ }^{17}$ Recasting the AR-model in state space form and treating the parameters as unobservables, it is possible to obtain a set of parameters for each point in time. These time-dependent AR-parameters can then be used to derive a time dependent spectrum. For details see the Appendix, Section A.3.2.
} 
Table 4: Changes in the Structure of the Real Wage and the Business Cycle

\begin{tabular}{lcccr}
\hline \hline & & \multicolumn{3}{c}{ Average Proportion of Total Variance } \\
& Period & 7-10 years & $5-7$ years & $3-5$ years \\
\hline$Y$ & $1969-81$ & 0.09 & 0.39 & 0.41 \\
$N$ & $1981-90$ & 0.21 & 0.35 & 0.21 \\
& $1969-81$ & 0.13 & 0.31 & 0.49 \\
$A / C p$ & $1981-90$ & 0.10 & 0.46 & 0.29 \\
& $1969-81$ & 0.07 & 0.35 & 0.48 \\
$A / P p$ & $1981-90$ & 0.32 & 0.12 & 0.29 \\
& $1969-81$ & 0.08 & 0.26 & 0.43 \\
$W / C P$ & $1981-90$ & 0.13 & 0.29 & 0.32 \\
& $1969-81$ & 0.07 & 0.33 & 0.50 \\
$W / P p$ & $1981-90$ & 0.22 & 0.13 & 0.35 \\
& $1969-81$ & 0.28 & 0.26 & 0.40 \\
\hline Notes: (i) $A:$ average hourly earnings; $W:$ average hourly earnings excluding over- \\
time; $P p:$ producer price index; $C p:$ consumer price index; $Y:$ output; $N:$ employ- \\
ment; (ii) The above results are for the BKM filter only.
\end{tabular}

\section{Conclusions}

When viewed from the frequency domain, real wages are unequivocally procyclical. On average, the dominant frequency band coincides with observed U.S. business cycles. Through time, we find evidence of positive associations between the lengths of the dominant wage frequency bands and the duration of the business cycle. Relationships are more straightforward if production is used as the basis of measuring cyclical changes. There are contemporaneous co-movements between wages and the production cycle. With an employment-based measure of the cycle, wages continue to display procyclicality but there are significant phase shifts between employment and wages. Our general finding of wage procyclicality is not, unlike time domain studies, dependent on the choice of wage deflator. We observe some differences between the use of a consumer price index and a producer price index in the context of Table 4 . With the former deflator, there appears to be more sensitivity between the changing structures of the business cycle and the wage.

We began by emphasising the simple point that there will exist a number of major cyclical influences, of differing strengths and amplitudes, that act upon the real wage. Frequency domain methodology does not lose sight of this observation. While, we have drawn particular attention to the dominant frequency band, we also show that other bands have also played 
important roles in determining the overall variation in real wage movements. Hopefully, this form of decomposition may help to enrich the study of the cycle in alternative economic applications. 


\section{A Appendix}

\section{A.1 Cosine/Sine Waves}

Consider the wave

$$
y_{t}=r^{t}\left(a_{1} \cos \omega t+a_{2} \sin \omega t\right) .
$$

Setting $a_{1}=A \cos B$ and $a_{2}=A \sin B$, this expression can be rewritten to obtain

$$
\begin{aligned}
y_{t} & =r^{t}\left(a_{1} \cos \omega t+a_{2} \sin \omega t\right)= \\
& =r^{t}(A \cos B \cos \omega t+A \sin B \sin \omega t)= \\
& =r^{t}(A \cos (\omega t-B)),
\end{aligned}
$$

with

$$
A=\sqrt{a_{1}^{2}+a_{2}^{2}} ; B=-\arctan \frac{a_{2}}{a_{1}}
$$

Interpretation:

Figure 5: $y_{t}=\cos (t)$

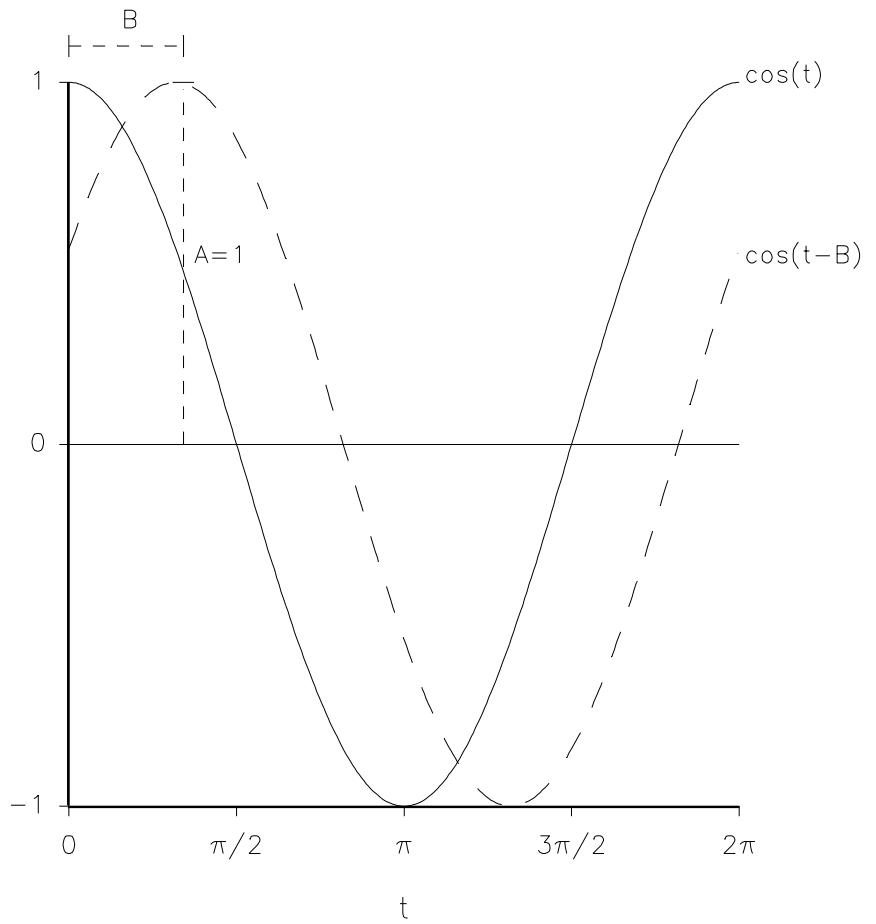

- Frequency and Period:

The length of a cycle is called period; the frequency measures how often a cycle is 
repeated during one unit of time. The functions $\cos (t)$ and $\sin (t)$ have the basic period $2 \pi$ and the frequency $1 / 2 \pi$. The function $\cos (\omega t)$ has the period $P=2 \pi / \omega$ and the frequency $\lambda=1 / P=\omega / 2 \pi$.

- Phase shift:

The function $\cos (\omega t-B)$ reaches its turning points $B / \omega$ time units later than $\cos (\omega t)$.

- Amplitude:

Since the range of the fluctuations of the function $\cos (\omega t-B)$ lies between -1 and 1 , the factor $A$ leads to a monotonic change of the amplitude.

- Modulus:

$r^{t}$ changes the amplitude; the change depends on the size of $r$.

- $r=1$ leads to a cycle with constant amplitude (uniform fluctuation).

$-r>1$ leads to a cycle with increasing amplitude (explosive fluctuation).

$-r<1$ leads to a cycle with decreasing amplitude (convergence); this is a stable solution.

The modulus measures the percentage change of the amplitude after $t$ units of time.

\section{A.2 Modified Baxter-King Filter}

Baxter and King (1995) construct a bandpass filter of finite order $K$ which is optimal in the sense that it is an approximate bandpass filter with trend-reducing properties and symmetric weights, which ensure that there is no phase shift in the filter output. In time domain, the impact of the filter on an input series $y_{t}$ is given by the finite moving average ${ }^{18} \tilde{y}_{t}=$ $\sum_{j=-K}^{K} a_{j} L^{j} y_{t}$. In the frequency domain, the filter is characterised by its Fourier transform $\alpha(\omega) .{ }^{19}$ To find the weights $a_{j}$, one solves the minimisation problem

$$
\min _{a_{j}} Q=\int_{-\pi}^{\pi}|\beta(\omega)-\alpha(\omega)|^{2} d \omega, \text { s.t. } \alpha(0)=0
$$

where $|\beta(\omega)|$ is the "ideal" filter gain with cut-off frequencies $\omega_{1}$ and $\omega_{2} \cdot{ }^{20}$ The constraint ensures that the resulting filter has trend reducing properties. ${ }^{21}$

\footnotetext{
${ }^{18} L$ denotes the backshift operator $\left(L^{n} y_{t}=y_{t-n}\right)$.

${ }^{19}$ See e.g. Koopmans (1974), p. $165 \mathrm{ff}$.

${ }^{20}$ The gain of a filter measures the change in the amplitude of the input components if transformed by the filter. The ideal bandpass filter gain $|\beta(\omega)|$ takes the value 1 in the frequency interval $\left[\omega_{1}, \omega_{2}\right]$ and 0 outside this interval.

${ }^{21}$ In order to remove the component with the frequency $\omega=0$ from the series, the filter weights must sum to zero (Baxter and King 1995, p. 26).
} 
Solving the minimisation problem leads to the following results: ${ }^{22}$

$$
\begin{aligned}
a_{j} & =b_{j}+\theta ; j=0, \pm 1, \ldots, \pm K ; \\
b_{j} & =\left\{\begin{array}{ll}
\frac{\omega_{2}-\omega_{1}}{\pi} & \text { if } j=0 \\
\frac{1}{\pi j}\left(\sin \omega_{2} j-\sin \omega_{1} j\right) & \text { if } j= \pm 1, \pm 2, \ldots
\end{array} ;\right. \\
\theta & =\frac{-\sum_{j=-K}^{K} b_{j}}{2 K+1} ;
\end{aligned}
$$

The original Baxter-King filter has an undesireable property, which is known as Gibb's phenomenon, due to the fact that the ideal filter, which is a discontinuous function of $\omega$, is approximated by a finite Fourier series. This approximation leads to side lobes in the gain function of the filter. (Priestley 1981, p. 561-3, Koopmans 1974, p. 187-9). While the relative contribution of some components for the overall variance of the series is exaggerated (i.e. they are multiplied by a gain greater than 1), other components are suppressed (i.e. they are multiplied by a gain less than 1).

An obvious solution to this problem is to increase the filter length. But since we are restricted by the limited availability of economic data, there is not much to be gained from changing the length of the filter. A more appropriate solution is to apply spectral windows. As an example, consider the so called Lanczos's $\sigma$ factors (Bloomfield 1976, p. 129-137). We replace the truncated weights of the optimal filter $b_{j}$ in equation (17) by the modified weights $b_{j}^{\star}$, which are obtained from

$$
b_{j}^{\star}=b_{j} \frac{\sin ((2 \pi j) /(2 K+1))}{(2 \pi j) /(2 K+1)} ;|j|=1, \ldots, K .
$$

After this step, the modified filter weights of the Baxter-King filter $a_{j}^{\star}$ can be calculated as demonstrated above (Woitek, 1998).

\section{A.3 Estimation of the Spectrum}

\section{A.3.1 Autoregressive Spectra}

To estimate the spectra, we fit autoregressive models in the time domain, and calculate the spectra of the estimated models. This method is based on the seminal work by Burg (1967), who shows that the resulting spectrum is formally identical to a spectrum derived on the Maximum Entropy Principle. This is seen to be a more reasonable approach then the normally used periodogram estimator. The periodogram employs the assumption that all the covariances outside the sample period are zero. Given that economic time series are

\footnotetext{
${ }^{22}$ The filter is symmetric (i.e. $a_{j}=a_{-j}$ ), and therefore does not impose a phase shift on the output.
} 
notoriously short, this seems to be a problematic assumption ${ }^{23}$ Consider a univariate AR model of order $p$, with residual variance $\sigma^{2}$. The spectrum is given by

$$
f(\omega)=\frac{1}{2 \pi} \frac{\sigma^{2}}{\left|1-\sum_{j=1}^{p} \alpha_{j} e^{-i \omega j}\right|^{2}} ; \omega \in[-\pi, \pi] .
$$

With a VAR model of order $p$, the spectral density matrix is given by

$$
\mathbf{F}(\omega)=\frac{1}{2 \pi} \mathbf{A}(\omega)^{-1} \Sigma \mathbf{A}(\omega)^{-\star} ; \omega \in[-\pi, \pi]
$$

$\boldsymbol{\Sigma}$ is the error variance-covariance matrix of the model, and $\boldsymbol{A}(\omega)$ is the Fourier transform of the matrix lag polynomial $\mathbf{A}(L)=\mathbf{I}-\mathbf{A}_{1} L-\cdots-\mathbf{A}_{p} L^{p} \cdot{ }^{24}$

\section{A.3.2 Time Dependent Spectra}

An important issue in the analysis of business cycles is the potential for the relationships under study to change over time. In the context of our methodology, this issue can be addressed by making the spectrum time dependent,

$$
f(\omega)_{t}=\frac{1}{2 \pi} \frac{\sigma_{t}^{2}}{\left|1-\sum_{j=1}^{p} \alpha_{j, t} e^{-i \omega j}\right|^{2}} ; \omega \in[-\pi, \pi] .
$$

To obtain a set of AR parameters for each point in time, we transform an AR model of order $p$ into state space form, treating the parameters as unobservable state vector:

$$
\begin{aligned}
& x_{t}=\left(\begin{array}{llll}
x_{t-1} & x_{t-2} & \ldots & x_{t-p}
\end{array}\right) \underbrace{\left(\begin{array}{c}
a_{1, t} \\
a_{2, t} \\
\vdots \\
a_{p, t}
\end{array}\right)}_{\mathbf{a}_{t}}+\epsilon_{t}, \\
& \mathbf{a}_{t}=\overline{\mathbf{a}}+\mathbf{T} \mathbf{a}_{t-1}+\boldsymbol{\zeta}_{t} .
\end{aligned}
$$

The errors $\epsilon_{t}$ and $\boldsymbol{\zeta}_{t}$ are assumed to be serially uncorrelated with variances $\sigma^{2}$ and $Q_{t}$, respectively. The transition matrix $\mathbf{T}_{t}$ is assumed to be a diagonal matrix. The value of the elements on the diagonal is 0.9 . Thus, the parameters follow a stationary AR(1) process. The parameters of the model are estimated using the Kalman filter. ${ }^{25}$

\footnotetext{
${ }^{23}$ See the discussion in (Priestley, 1981, p. 432, 604-607). A recent applications to economic time series is A'Hearn and Woitek (2001).

${ }^{24} \mathrm{~L}$ is the backshift operator; the superscript ' ' denotes the complex conjugate transpose.

${ }^{25}$ For details, see Harvey (1992).
} 


\section{A.4 Figures and Tables}

Figure 6: Real Wages and Industrial Production: Phase Shift, BKM Filter (1)
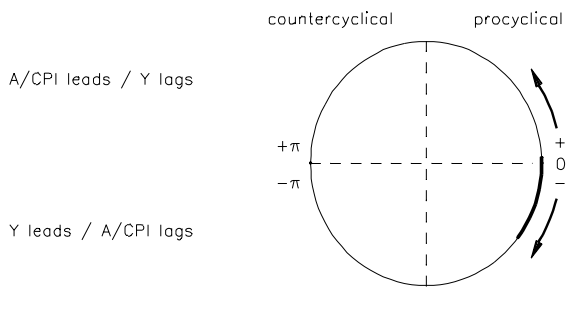

Period 5.41

heods / A/CPl logs

Rel. Phose Shift $\quad-0.02$

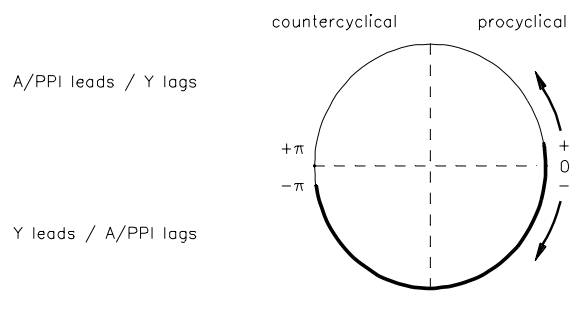

$\begin{array}{lc}\text { Period } & 5.78 \\ \text { Rel. Phase Shift } & -0.06\end{array}$
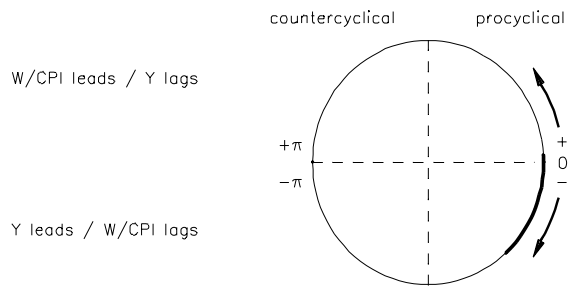

$\begin{array}{lc}\text { Period } & 5.35 \\ \text { Rel. Phose Shift } & -0.03\end{array}$

W/PPI leads / Y lags

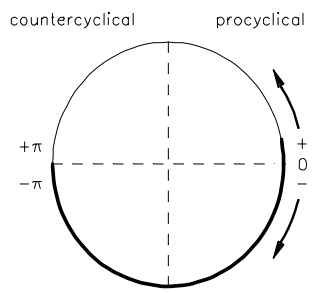

$\begin{array}{lr}\text { Period } & 5.73 \\ \text { Rel. Phose Shift } & -0.09\end{array}$ 
Figure 7: Real Wages and Employment: Phase Shift, BKM Filter (1)

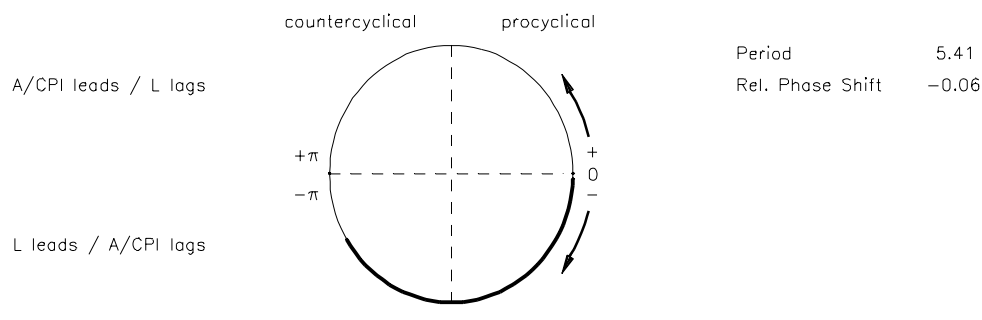

A/PPI leods / L lags

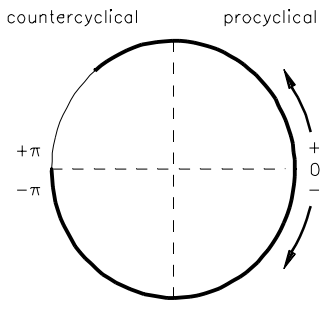

Period 5.78

Rel. Phose Shift

Lleads / A/PPI lags

$\begin{array}{lr}\text { Period } & 5.41 \\ \text { Rel. Phase Shift } & -0.06\end{array}$

P.

W/CPI leads / L lags

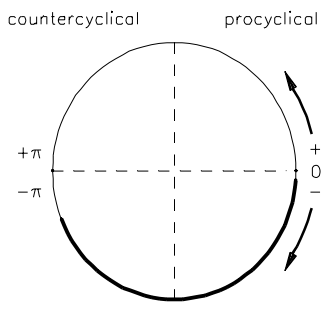

$\begin{array}{lr}\text { Period } & 5.35 \\ \text { Rel. Phose Shift } & -0.08\end{array}$

L leads / W/CPI lags

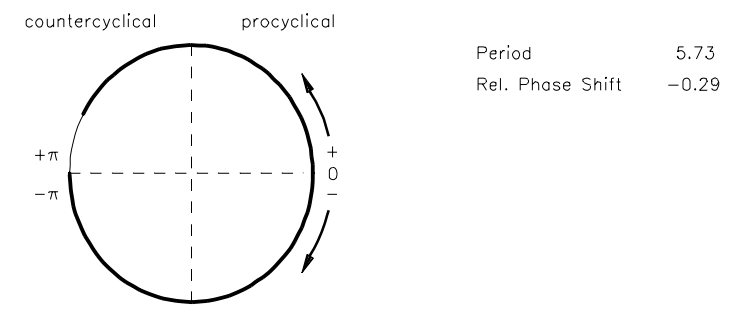

$\begin{array}{lr}\text { Period } & 5.73 \\ \text { Rel. Phose Shift } & -0.29\end{array}$

W/PPI leads / L lags

L leads / W/PPI lags 


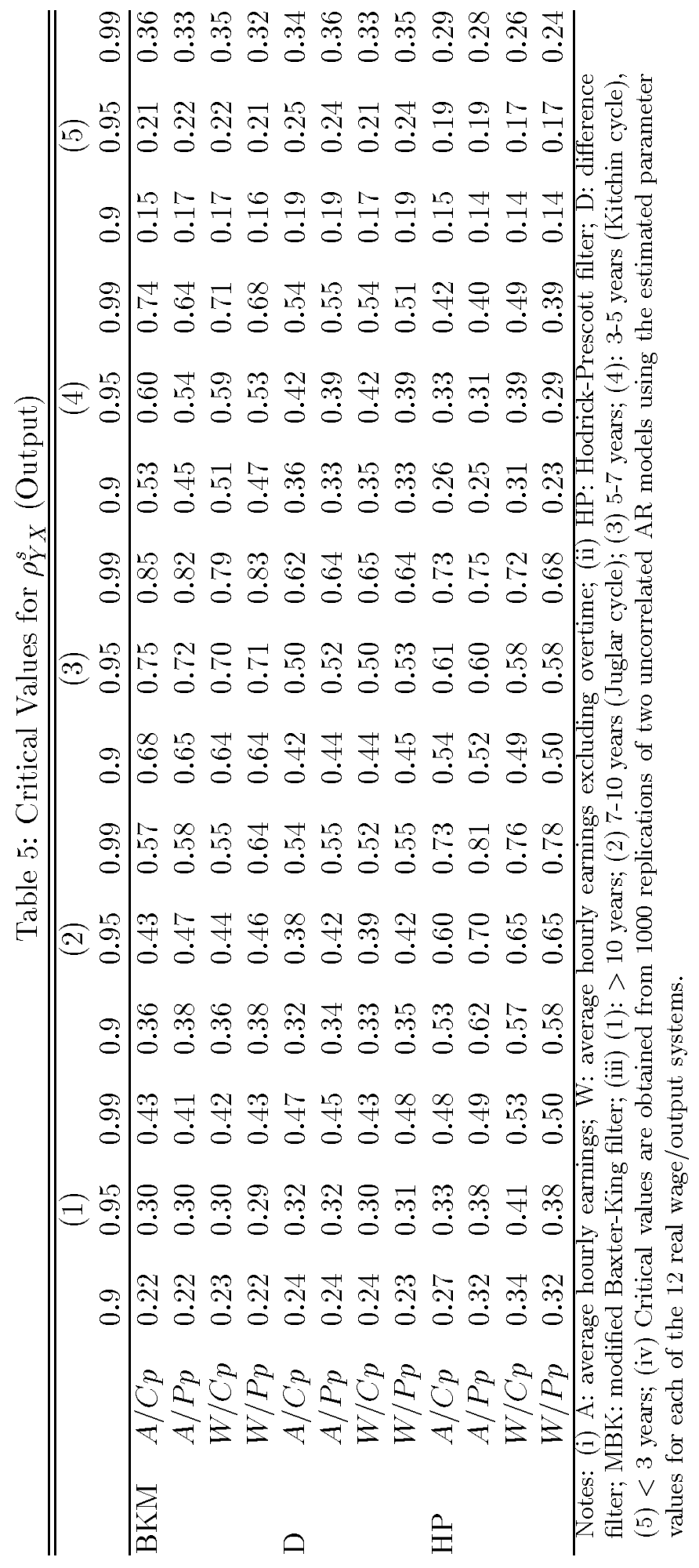




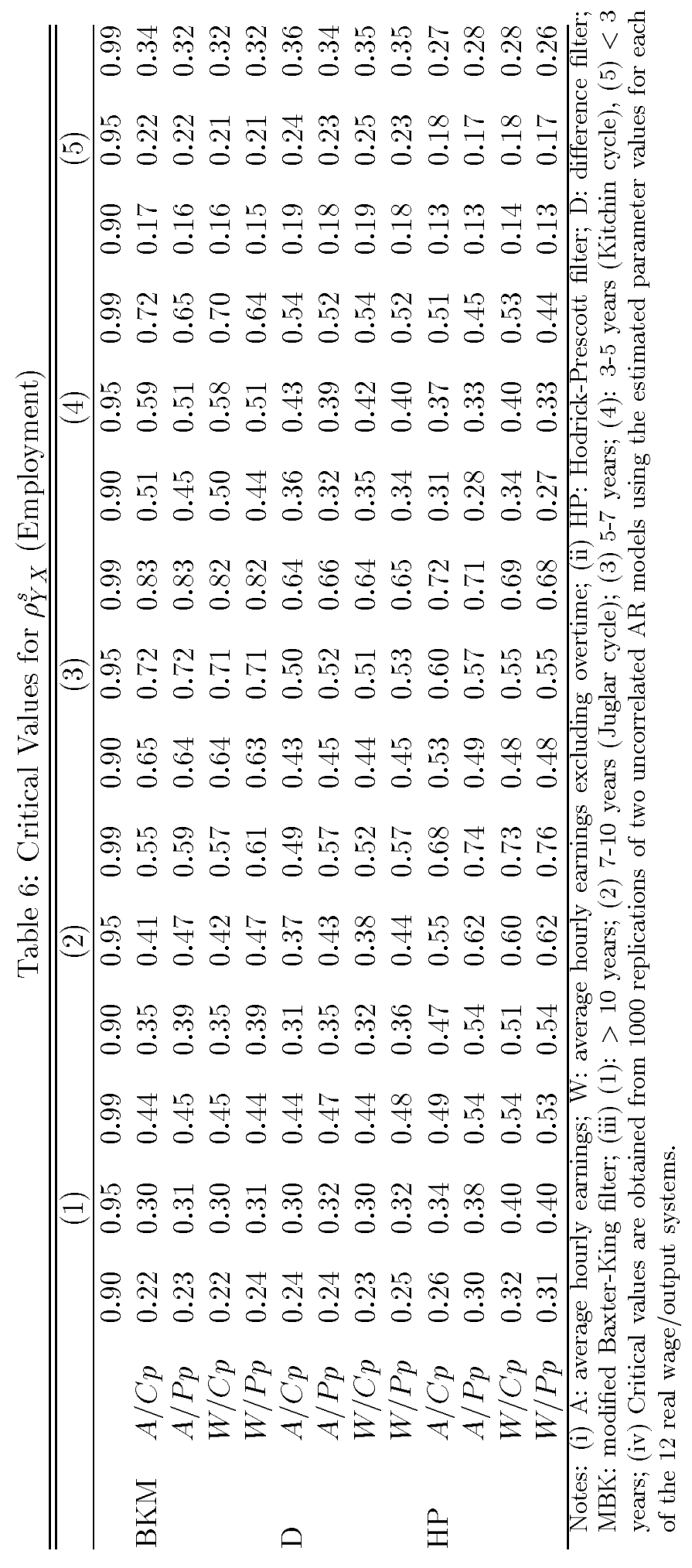




\section{References}

Abraham, K. G. and Haltiwanger, J. C. (1995), "Real Wages and the Business Cycle." Journal of Economic Literature XXXIII, 1215-1264.

A'Hearn, B. and Woitek, U. (2001), "More International Evidence on the Historical Properties of Business Cycles." Journal of Monetary Economics .

Backus, D. K. and Kehoe, P. J. (1992), "International Evidence on the Historical Properties of Business Cycles." American Economic Review 82, 864-888.

Baxter, M. and King, R. G. (1995), "Measuring Business Cycles. Approximate Band-Pass Filters for Economic Time Series.", NBER Working Paper Series, No. 5022.

Bloomfield, P. (1976), Fourier Analysis of Time Series: An Introduction. New York, London, Sidney, Toronto: John Wiley \& Sons.

Burg, J. P. (1967), "Maximum Entropy Spectral Analysis." In: Proceedings of the 37th Meeting of the Society of Exploration Geophysicists, reprinted in Childers, D. G. (1978), Modern Spectrum Analysis. New York: IEEE Press.

Cogley, T. and Nason, J. M. (1995), "Effects of the Hodrick-Prescott Filter on Trend and Difference Stationary Time Series: Implications for Business Cycle Research." Journal of Economic Dynamics and Control 19, 253-278.

Efron, B. and Tibshirani, R. J. (1993), An Introduction to the Bootstrap. New York: Chapman \& Hall.

Hamermesh, D. S. (1993), Labor Demand. Princeton: Princeton University Press.

Hart, R. A. and Malley, J. R. (2001), "On the Cyclicality and Stability of Real Earnings.", University of Glasgow, mimeo.

Harvey, A. C. (1992), Forecasting, Structural Time Series Models and the Kalman Filter. Cambridge: Cambridge University Press.

Harvey, A. C. and Jaeger, A. (1993), "Detrending, Stylized Facts and the Business Cycle." Journal of Econometrics 8, 231-247.

Hodrick, R. and Prescott, E. (1980), "Postwar U.S. Business Cycles: An Empirical Investigation.", Discussion Paper No. 451, Carnegie-Mellon University.

King, R. G. and Rebelo, S. T. (1993), "Low Frequency Filtering and Real Business Cycles." Journal of Economic Dynamics and Control 17, 207-231. 
Koopmans, L. H. (1974), The Spectral Analysis of Time Series. New York, San Francisco, London: Academic Press.

Kydland, F. E. and Prescott, E. C. (1990), "Business Cycles: Real Facts and A Monetary Myth." Federal Reserve Bank of Minneapolis, Quarterly Review 14, 3-18.

Priestley, M. (1981), Spectral Analysis and Time Series. London: Academic Press.

Woitek, U. (1998), "A Note on the Baxter-King Filter.", University of Glasgow, Discussion Papers in Economics, No 9813. 